PHYSICAL REVIEW RESEARCH 3, L032041 (2021)

\title{
Power fluctuations in a finite-time quantum Carnot engine
}

\author{
Tobias Denzler $\odot$ and Eric Lutz $\odot$ \\ Institute for Theoretical Physics I, University of Stuttgart, D-70550 Stuttgart, Germany
}

(Received 13 July 2020; accepted 13 July 2021; published 13 August 2021)

\begin{abstract}
Stability is an important property of small thermal machines with fluctuating power output. We here consider a finite-time quantum Carnot engine based on a degenerate multilevel system and study the influence of its finite Hilbert space structure on its stability. We optimize in particular its relative work fluctuations with respect to level degeneracy and level number. We find that its optimal performance may surpass those of nondegenerate two-level engines or harmonic oscillator motors. Our results show how to realize high-performance, high-stability cyclic quantum heat engines.
\end{abstract}

DOI: 10.1103/PhysRevResearch.3.L032041

The Carnot engine is one of the most emblematic examples of a thermal machine. Since its introduction in 1824, it has become a representative model for all heat engines. The Carnot cycle simply consists of two isothermal (expansion and compression) steps and of two adiabatic (expansion and compression) processes [1]. In its ideal reversible limit of infinite cycle duration, the Carnot motor is the most efficient heat engine. The corresponding Carnot efficiency, $\eta_{\mathrm{C}}=1-T_{c} / T_{h}$, where $T_{c, h}$ are the respective temperatures of the cold and hot heat baths, is regarded as the first formulation of the second law of thermodynamics [1]. The first experimental realization of a Carnot engine using a colloidal particle in an optical harmonic trap has been reported lately [2]. In addition, the finite-time properties of the Carnot cycle have been well investigated theoretically both in the classical [3-10] and in the quantum [11-19] regimes. Strong emphasis has been put on the optimization of the performance of the engine for finite cycle durations, in particular on its average power output at the expense of its efficiency.

For classical microscopic heat engines, such as the one implemented in the experiment of Ref. [2], thermal fluctuations are no longer negligible as is the case for macroscopic motors [20]. As a result, key performance measures, such as efficiency [21-24] and power [25-28], are stochastic variables. In that context, attention has recently been drawn to power fluctuations as a limiting factor for the practical usefulness of thermal machines: Heat engines should indeed ideally have high-efficiency, large power output but small power fluctuations [25-28]. A new figure of merit, the constancy, defined as the product of the variance of the power and time, has been introduced to characterize the stability of heat engines [25-28]. While a strict trade-off between efficiency, power,

Published by the American Physical Society under the terms of the Creative Commons Attribution 4.0 International license. Further distribution of this work must maintain attribution to the author(s) and the published article's title, journal citation, and DOI. and constancy has been established for steady-state heat engines, implying that power fluctuations diverge at maximum efficiency and finite power [27], they may remain finite for quasistatic cyclic thermal machines [28]. On the other hand, quantum motors are not only dominated by thermal fluctuations but also by quantum fluctuations.

In this Letter, we investigate the generic features of the power fluctuations in a finite-time quantum Carnot engine in the quasistatic limit. We specifically study the interplay between power fluctuations, the finite dimensionality of the Hilbert space of the working medium, and the degree of degeneracy of its levels. Degenerate finite level structures commonly appear in atomic [29], molecular [30], and condensed-matter physics [31]. An understanding of their influence on the stability of quantum heat engines is therefore essential for future experimental realizations of thermal devices in these systems [32]. An important illustration of the effect of the finiteness of quantum systems on thermodynamic fluctuations is provided by the Schottky anomaly [33]: The corresponding increase of the heat capacity at low temperatures does not occur in infinite-dimensional systems such as the harmonic oscillator, in which the energy is not bounded; it is, furthermore, strongly affected by level degeneracy and level number [34]. We mention, however, that our results are not directly related to the Schottky anomaly.

In the following, we compute the inverse coefficient of variation for work, defined as the ratio of the mean work and its standard deviation [35], for a quasistatic quantum Carnot engine whose working medium is described by a homogeneous Hamiltonian of degree $-2, \mathcal{H}(b \boldsymbol{r})=b^{-2} \mathcal{H}(\boldsymbol{r})$. Such Hamiltonians characterize a large class of single-particle, many-body, and nonlinear systems that exhibit equidistant spectra [36-40]. We obtain a general formula that only depends on the heat capacity and on the entropy variation during the hot isotherm. We use this expression to maximize the inverse coefficient of variation for work, with respect to the degree of degeneracy and the number of levels of the system, in order to attain optimal cyclic quantum engines that operate close to the Carnot efficiency with large power output and small power fluctuations. We illustrate our results by 
analyzing (i) a two-level system with arbitrary degeneracy, (ii) a nondegenerate system with an arbitrary level number, and (iii) a three-level system with a generic degree of degeneracy. In all cases the optimal inverse coefficient of variation for work may be numerically determined by solving a transcendental equation.

Finite-time quantum Carnot cycle. We consider a general quantum system with a time-dependent Hamiltonian, $H_{t}=$ $\omega_{t} \mathcal{H}$, as the working fluid of a finite-time quantum Carnot engine, with driving parameter $\omega_{t}$. The quantum Carnot cycle consists of the following four steps [11-19]: (1) hot isothermal expansion from $\omega_{1}$ to $\omega_{2}$, at temperature $T_{h}$ in time $\tau_{1}$, during which work $W_{1}$ is produced by the system and heat $Q_{h}$ is absorbed; (2) adiabatic expansion from $\omega_{2}$ to $\omega_{3}$, in time $\tau_{2}$, during which work $W_{2}$ is performed and the entropy remains constant. The system is here decoupled from the baths and its Hamiltonian commutes with itself at all times, $\left[H_{t}, H_{t^{\prime}}\right]=0$. As a result, nonadiabatic transitions do not occur for all driving times $\tau_{2}$; (3) cold isothermal compression from $\omega_{3}$ to $\omega_{4}$, at temperature $T_{c}$ in time $\tau_{3}$, during which work $W_{3}$ is done on the system and heat $Q_{c}$ is released; (4) adiabatic compression from $\omega_{4}$ to $\omega_{1}$, in time $\tau_{4}$, during which work $W_{4}$ is unitarily performed on the system. The total cycle time is $\tau=\sum_{i} \tau_{i}$. Work and heat are taken positive when added to the system.

In order to evaluate the finite-time performance of the quantum Carnot engine, we will determine the mean and the variance of the stochastic work output $w$. The average total work, $W=\langle w\rangle=\sum_{i} W_{i}$, directly follows from the combination of the first and second law [1],

$$
W=-Q_{h}-Q_{c}=\left(T_{c}-T_{h}\right) \Delta S,
$$

where $\Delta S$ denotes the entropy change during the hot isotherm. Meanwhile, the total work fluctuations are characterized by the probability distribution $P(w)$,

$$
P(w)=\left\langle\delta\left(w-\sum_{i} w_{i}\right)\right\rangle,
$$

where the average is taken over the joint probability distribution of the work of the four branches of the cycle, $P\left(w_{1}, w_{2}, w_{3}, w_{4}\right)$ [28]. The two isotherms (1) and (3) are assumed to be slower than the (fast) relaxation induced by the baths. The system thus remains in a thermal state and the two finite-time processes are quasistatic. In this case, the work distributions are sharp (with no fluctuations) and work is deterministic [41],

$$
P_{1,3}\left(w_{1,3}\right)=\delta\left(w_{1,3}-W_{1,3}\right) .
$$

On the other hand, since no heat is exchanged during the two unitary adiabats (2) and (4), the corresponding work distributions can be obtained via the usual two-point-measurement scheme by projectively measuring the energy at the beginning and at the end of each step [42]. Without level transitions, we obtain for process (2),

$$
P_{2}\left(w_{2}\right)=\sum_{n} \delta\left[w_{2}-\left(E_{3}^{n}-E_{2}^{n}\right)\right] p_{2}^{n},
$$

where $E_{2}^{n}$ and $E_{3}^{n}$ denote the respective eigenvalues of the Hamiltonians $H_{2}=H_{\tau_{1}}$ and $H_{3}=H_{\tau_{1}+\tau_{2}}$. The initial thermal distribution reads $p_{2}^{n}=\exp \left(-\beta_{h} E_{2}^{n}\right) / z_{2}$ with inverse hot temperature $\beta_{h}$ and partition function $z_{2}$. We have similarly for transformation (4),

$$
P_{4}\left(w_{4}\right)=\sum_{m} \delta\left[w_{4}-\left(E_{1}^{m}-E_{4}^{m}\right)\right] p_{4}^{m},
$$

with $p_{4}^{m}=\exp \left(-\beta_{c} E_{4}^{m}\right) / z_{4}$. In order to ensure that the system is in a thermal state at the end of each adiabat, and thus at the beginning of each isotherm, we adjust the adiabatic driving such that $\omega_{3} / \omega_{2}=\omega_{4} / \omega_{1}=\beta_{h} / \beta_{c}$ [18]. The whole Carnot cycle is hence quasistatic.

Combining the contributions of all the four branches of the cycle, we find the work output distribution,

$$
P(w)=\left\langle\delta\left[w-\left(W-\widetilde{\Delta H_{2}}-\widetilde{\Delta H}_{4}\right)\right]\right\rangle,
$$

where $W$ is given by Eq. (1). We have furthermore defined the (stochastic) difference from the mean $\widetilde{\Delta H}_{i}=\left\langle\Delta H_{i}\right\rangle-\Delta H_{i}$, where $\Delta H_{i}$ is the energy change during adiabat $i$, and used the cycle condition $\sum_{i}\left\langle\Delta H_{i}\right\rangle=0$. The average in Eq. (6) may be computed using the Boltzmann distributions at the beginning of each adiabat [41].

Coefficient of variation for work. In statistics, the Fano factor (the ratio of the variance $\sigma^{2}$ and the mean) and the coefficient of variation (the ratio of the standard deviation $\sigma$ and the mean) are two measures of the dispersion of a probability distribution [35]. For heat engines, the Fano factor for work, $\sigma_{w}^{2} / W$, is equal to the quotient of the constancy $\sigma_{P}^{2} \tau$ and the average power $P=W / \tau$ (defined over one cycle time) [28], while the corresponding coefficient of variation for work describes the relative work fluctuations. All the moments of the total work can be evaluated from Eq. (6) by integration $\left\langle w^{n}\right\rangle=\int d w P(w) w^{n}$. The variance then reads [41]

$$
\sigma_{w}^{2}=\left(T_{c}-T_{h}\right)^{2}\left[C\left(\beta_{h}, \omega_{2}\right)+C\left(\beta_{c}, \omega_{4}\right)\right],
$$

where we have introduced the heat capacity of the system, $C\left(\beta_{j}, \omega_{i}\right)=d\left\langle H_{i}\right\rangle / d T_{j}$, at the beginning of each adiabat [41]. We accordingly obtain the Fano factor,

$$
\frac{\sigma_{w}^{2}}{|W|}=\frac{\left(T_{h}-T_{c}\right)\left[C\left(\beta_{h}, \omega_{2}\right)+C\left(\beta_{c}, \omega_{4}\right)\right]}{\Delta S},
$$

and the corresponding coefficient of variation,

$$
\frac{\sigma_{w}}{|W|}=\frac{\sqrt{C\left(\beta_{h}, \omega_{2}\right)+C\left(\beta_{c}, \omega_{4}\right)}}{\Delta S} .
$$

Equations (8) and (9) describe similar physics. However, in contrast to the Fano factor (8), the coefficient of variation (9) has the advantage that (i) it is a dimensionless quantity that (ii) depends solely on the heat capacities of the system (since the entropy variation can be written as an integral of the heat capacities [41]). We shall therefore focus on that quantity in the following.

A finite-time quantum Carnot engine with large work output and small work output fluctuations is characterized by a large inverse coefficient of variation $|W| / \sigma_{w}$. We will thus next optimize the inverse of Eq. (9) with respect to the degree of degeneracy and with respect to the number of levels of the working medium.

Degenerate two-level system. We begin by considering a degenerate qubit with Hamiltonian $H_{t}=\omega_{t} g_{1}|1\rangle\langle 1|$, where 


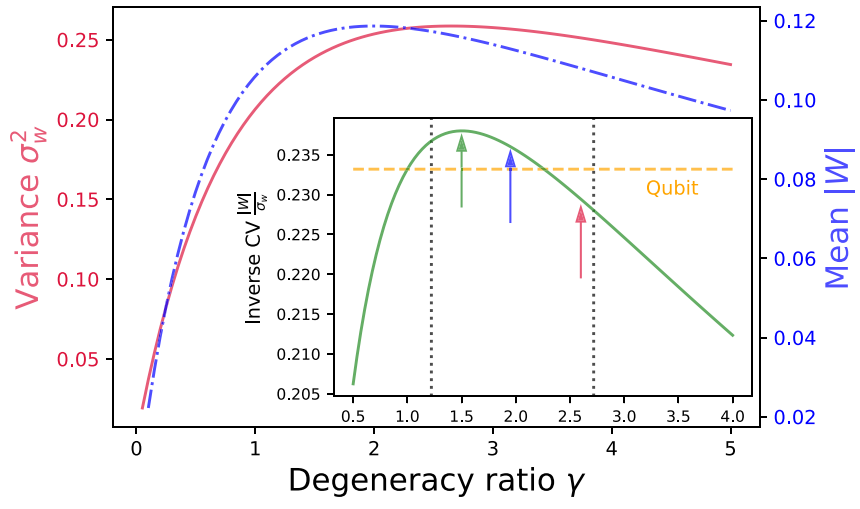

FIG. 1. Average work output $|W|$, Eq. (1) (blue dashed-dotted), its variance $\sigma_{w}^{2}$, Eq. (7) (red), and inverse coefficient of variation (COV), $|W| / \sigma_{w}$, Eq. (9) (green, inset), for a degenerate two-level quantum Carnot engine, as a function of the degeneracy ratio $\gamma$. The maximum COV (green arrow) outperforms its nondegenerate counterpart (orange dashed line). The blue (red) arrow indicates the maximum value of the average (variance) of the work output. The vertical black dotted lines mark the respective maxima of the heat capacity (Schottky anomaly), Eq. (10), at the hot and cold bath temperatures. Parameters are $\beta_{c}=1, \beta_{h}=0.1, \omega_{2}=2$, and $\omega_{4}=1$.

$g_{0}$ and $g_{1}$ are the respective degeneracies of the ground $|0\rangle$ and excited $|1\rangle$ states. The partition function at inverse temperature $\beta$ and frequency $\omega$ is $Z_{2}=g_{0}+g_{1} \exp (-\beta \omega)$ [33]. The heat capacity then follows as

$$
C_{2}(\beta, \omega)=\frac{\gamma(\beta \omega)^{2}}{\left(e^{\beta \omega / 2}+\gamma e^{-\beta \omega / 2}\right)^{2}},
$$

with the degeneracy ratio $\gamma=g_{1} / g_{0}$. The entropy difference during the hot isotherm further reads

$$
\Delta S_{2}=\frac{\beta_{c} \omega_{4}}{\gamma^{-1} e^{\beta_{c} \omega_{4}}+1}-\frac{\beta_{h} \omega_{2}}{\gamma^{-1} e^{\beta_{h} \omega_{2}}+1}+\ln \left[\frac{1+\gamma e^{-\beta_{h} \omega_{2}}}{1+\gamma e^{-\beta_{c} \omega_{4}}}\right] .
$$

The average of the work output (1) (blue dashed-dotted), its variance (7) (red), and the corresponding inverse coefficient of variation (9) (green, inset) are shown in Fig. 1 as a function of the degeneracy ratio $\gamma$. We observe that, for given frequencies and bath temperatures, both mean and variance first increase with increasing values of $\gamma$, before they both decrease as a result of the finiteness of the Hilbert space of the qubit. However, the mean augments and decays faster than the variance. As a consequence, the inverse coefficient of variation for work exhibits a clear maximum (green arrow) for an optimal degeneracy value $\bar{\gamma}$. Remarkably, the degenerate quantum Carnot engine here outperforms its nondegenerate counterpart $(\gamma=1)$ (orange dashed). The optimal value of the degeneracy of the working medium may be determined by numerically solving the transcendental equation,

$$
\begin{aligned}
& \left(\frac{1}{2} \partial_{\gamma}-1\right)\left[C_{2}\left(\beta_{c}, \omega_{4}\right)+C_{2}\left(\beta_{h}, \omega_{2}\right)\right] \\
& \quad+\left(\frac{1}{2}-\partial_{\gamma}\right) \Delta S_{2}=0 .
\end{aligned}
$$

The existence of such an optimal solution is guaranteed by continuity and the limiting behaviors at $\gamma \rightarrow 0$ and $\gamma \rightarrow \infty$, where the inverse coefficient of variation vanishes.

Two additional features are worth emphasizing. First, the conditions of minimal relative work fluctuations and of maximum work output (blue arrow) corresponding to

$$
\bar{\gamma}_{\mathrm{work}}=\frac{2\left(e^{2 x+y}-e^{x+2 y}-e^{x+2 y} x+e^{2 x+y} y\right)}{e^{2 x}-e^{2 y}+2 e^{x+y}(x-y)+\left(e^{x}-e^{y}\right) f(x, y)},
$$

with the variables $x=\beta_{c} \omega_{4}$ and $y=\beta_{h} \omega_{2}$, and $f(x, y)=$ $\sqrt{e^{2 x}+e^{2 y}-2 e^{x+y}+4 x y e^{x+y}}$, lead to two different solutions. While level degeneracy may be used to boost the work output [43-48], this enhancement is accompanied by an increase of work fluctuations, that is, of the instability of the machine. This property might be detrimental for practical implementations of quantum heat engines. On the other hand, the point of the maximum inverse coefficient of variation for work leads to an overall smaller work output but to a more stable engine. In addition, we note that the optimal value $\bar{\gamma}$ is bounded by the degeneracies associated with the respective maxima of the heat capacities (Schottky anomaly) at the hot and cold temperatures (vertical black dotted lines in Fig. 1),

$$
e^{\beta_{h} \omega_{2}} \leqslant \bar{\gamma} \leqslant e^{\beta_{c} \omega_{4}} .
$$

These bounds get tight when the limiting engine condition $\omega_{4} \beta_{c} \geqslant \omega_{2} \beta_{h}$ is approached.

Nondegenerate $N$-level system. In order to investigate the influence of the level number of the working fluid on the relative work fluctuations, we next examine a nondegenerate $N$-level system with equidistant spacing, $H_{t}=$ $\omega_{t} \sum_{n=0}^{N-1} n|n\rangle\langle n|$, as appearing in homogeneous Hamiltonians of degree -2 [36-40]. The partition function at inverse temperature $\beta$ and frequency $\omega$ is given by $Z_{N}=$ $[1-\exp (-N \beta \omega)] /[1-\exp (-\beta \omega)]$ [33]. The explicit (and lengthy) expressions for the heat capacity $C_{N}(\beta, \omega)$ and the entropy difference $\Delta S_{N}$ are given in the Supplemental Material [41]. Compact expressions for the inverse coefficient of variation for work may be obtained in the limit of a harmonic oscillator $(N \rightarrow \infty)$,

$$
\left(\frac{|W|}{\sigma_{w}}\right)_{\infty}=\frac{\Delta S_{\infty}}{\sqrt{[\operatorname{sech}(y / 2) y]^{2}+[\operatorname{sech}(x / 2) x]^{2}}},
$$

and for the case a (nondegenerate) qubit $(N=2)$,

$$
\left(\frac{|W|}{\sigma_{w}}\right)_{2}=\frac{\Delta S_{2}}{\sqrt{y^{2}\left[1-\tanh (y)^{2}\right]+x^{2}\left[1-\tanh (x)^{2}\right]}} .
$$

In the high-temperature limit, $\beta_{c, h} \omega_{4,2} \ll 1$, Eq. (15) reduces to the result obtained for the classical harmonic Carnot heat engine in Ref. [28],

$$
\left(\frac{|W|}{\sigma_{w}}\right)_{\infty}^{\mathrm{cl}}=\frac{\Delta S_{\infty}}{\sqrt{2}}
$$

On the other hand, the high-temperature limit of Eq. (16) exhibits a completely different $(x, y)$ dependence, which reflects the finite Hilbert space of the qubit,

$$
\left(\frac{|W|}{\sigma_{w}}\right)_{2}^{\mathrm{cl}}=\frac{\Delta S_{2}}{\sqrt{x^{2}\left[1-x^{2}\right]+y^{2}\left[1-y^{2}\right]}} .
$$




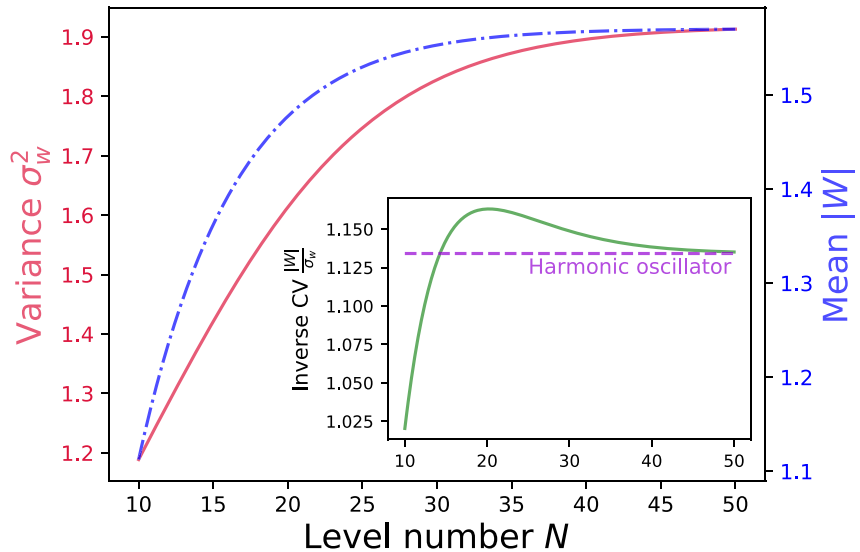

FIG. 2. Average work output $|W|$, Eq. (1) (blue dashed-dotted), its variance $\sigma_{w}^{2}$, Eq. (7) (red), and inverse coefficient of variation (COV), $|W| / \sigma_{w}$, Eq. (9) (green, inset), for a nondegenerate $N$-level quantum Carnot engine, as a function of the level number $N$. The maximum COV outperforms both that of the two-level engine and that of the harmonic oscillator motor (violet dashed line). Same parameters as in Fig. 1.

Such behavior can be traced back to the properties of the heat capacity in Eq. (9): While it reaches a constant value for the (infinite-dimensional) harmonic oscillator in the classical limit (Dulong-Petit law), it vanishes for the (finitedimensional) two-level system [33].

Figure 2 displays the mean work output $|W|$ (blue dasheddotted), the corresponding variance $\sigma_{w}^{2}$ (red), as well as the inverse coefficient of variation (9) (green, inset) as a function of the level number $N$. Mean and variance increase monotonously with $N$, reaching the respective values of the harmonic oscillator in the limit $N \rightarrow \infty$. However, the mean saturates faster than the variance. The inverse relative work fluctuations therefore present a maximum that outperforms both that of the two-level engine and of the harmonic oscillator motor. Contrary to naive expectation, the $N$-level Carnot engine does thus not simply interpolate between these two extreme situations. The point of maximum inverse coefficient of variation is again different from the point of maximum work output because of increased work fluctuations. The optimal level number $\bar{N}$ satisfies the transcendental equation,

$$
\begin{aligned}
& \left(\frac{1}{2} \partial_{N}-1\right)\left[C_{N}\left(\beta_{c}, \omega_{4}\right)+C_{N}\left(\beta_{h}, \omega_{2}\right)\right] \\
& +\left(\frac{1}{2}-\partial_{N}\right) \Delta S_{N}=0,
\end{aligned}
$$

which may be solved numerically.

Degenerate three-level system. We finally illustrate the usefulness of Eq. (9) for determining the maximum inverse coefficient of variation for work for degenerate multilevel quantum Carnot engines by treating the case of a degenerate three-level system with Hamiltonian $H_{t}=\omega_{t}\left(g_{1}|1\rangle\langle 1|+\right.$ $\left.2 g_{2}|2\rangle\langle 2|\right)$ and arbitrary level degeneracies $g_{n}(n=0,1,2)$. The partition function at inverse temperature $\beta$ and frequency $\omega$ is here $Z_{3}=g_{0}+g_{1} e^{-\beta \omega}+g_{2} e^{-2 \beta \omega}$. The corresponding inverse coefficient of variation for work (9) is represented as a function of the two degeneracy ratios $\gamma_{1}=g_{1} / g_{0}$ and

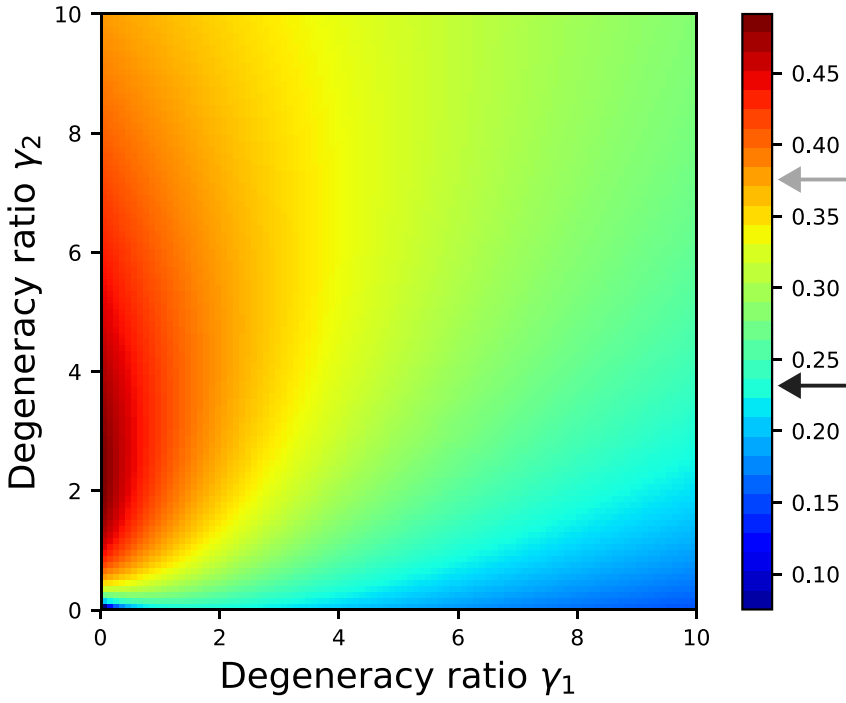

FIG. 3. Inverse coefficient of variation for work, $|W| / \sigma_{w}$, Eq. (9), for a degenerate three-level quantum Carnot engine, as a function of the degeneracy ratios $\gamma_{1}$ and $\gamma_{2}$. We notice a region of high inverse coefficient of variation for small $\gamma_{1}$ and $1 \lesssim \gamma_{2} \lesssim 4$, where nondegenerate two-level (black arrow) and three-level heat engines (gray arrow) are outperformed. Same parameters as in Fig. 1.

$\gamma_{2}=g_{2} / g_{0}$ in Fig. 3. We identify a region of high inverse coefficient of variation for small $\gamma_{1}$ and $1 \lesssim \gamma_{2} \lesssim 4$, where the quantum Carnot engine outperforms the respective nondegenerate two-level (black arrow) and three-level engines (gray arrow). We moreover notice that large ratios $\gamma_{1}$, that is, high degeneracy of the first level, is generally detrimental to the performance of the heat engine.

Conclusions. Two-level systems and harmonic oscillators have been the models of choice for the investigations of quantum heat engines in the past decades due to their simplicity [11-19]. Such finite-time engines have been mostly optimized by maximizing averaged performance measures, such as mean power, with respect to cycle duration, frequency, or temperature [11-19]. We have here extended these studies to include the effects of work fluctuations and of finite Hilbert space of the working medium, two essential features of small quantum machines. To this end, we have derived a compact expression of the relative work fluctuations of a finite-time quantum Carnot engine, as given by Eq. (9), in terms of the heat capacity of the system. We have shown that the quantum motor can outperform its nondegenerate counterparts, when optimized with respect to level degeneracy or level number. We have additionally found that optimizing the average work output, while ignoring work output fluctuations, generally leads to machines with larger instability. Our findings hence enable the analysis and future experimental realization of both high-performance and high-stability quantum engines.

We acknowledge financial support from the Volkswagen Foundation under project "Quantum coins and nano sensors" and the German Science Foundation (DFG) under Project No. FOR 2724 
[1] Y. A. Cengel and M. A. Boles, Thermodynamics. An Engineering Approach (McGraw-Hill, New York, 2001).

[2] I. A. Martinez, E. Roldan, L. Dinis, D. Petrov, J. M. R. Parrondo, and R. A. Rica, Brownian Carnot engine, Nat. Phys. 12, 67 (2015).

[3] F. L. Curzon and B. Ahlborn, Efficiency of a Carnot engine at maximum power output, Am. J. Phys. 43, 22 (1975).

[4] B. Andresen, R. S. Berry, A. Nitzan, and P. Salamon, Thermodynamics in finite time. I. The step-Carnot cycle, Phys. Rev. A 15, 2086 (1977).

[5] D. Gutkowicz-Krusin, I. Procaccia, and J. Ross, On the efficiency of rate processes. Power and efficiency of heat engines, J. Chem. Phys. 69, 3898 (1978).

[6] M. H. Rubin, Optimal configuration of a class of irreversible heat engines, Phys. Rev. A 19, 1277 (1979).

[7] P. Salamon and A. Nitzan, Finite time optimizations of a Newton's law Carnot cycle, J. Chem. Phys. 74, 3546 (1981).

[8] M. J. Ondrechen, M. H. Rubin, and Y. B. Band, The generalized Carnot cycle: A working fluid operating in finite time between finite heat sources and sinks, J. Chem. Phys. 78, 4721 (1983).

[9] M. Esposito, R. Kawai, K. Lindenberg, and C. Van den Broeck, Efficiency at Maximum Power of Low-Dissipation Carnot Engines, Phys. Rev. Lett. 105, 150603 (2010).

[10] V. Cavina, A. Mari, and V. Giovannetti, Slow Dynamics and Thermodynamics of Open Quantum Systems, Phys. Rev. Lett. 119, 050601 (2017).

[11] E. Geva and R. Kosloff, A quantum-mechanical heat engine operating in finite time. A model consisting of spin-1/2 systems as the working fluid, J. Chem. Phys. 96, 3054 (1992).

[12] E. Geva and R. Kosloff, On the classical limit of quantum thermodynamics in finite time, J. Chem. Phys. 97, 4398 (1992).

[13] F. Wu, L. Chen, S. Wu, F. Sun, and C. Wu, Performance of an irreversible quantum Carnot engine with spin 1/2, J. Chem. Phys. 124, 214702 (2006).

[14] H. T. Quan, Y. Liu, C. P. Sun, and F. Nori, Quantum thermodynamic cycles and quantum heat engines, Phys. Rev. E 76, 031105 (2007).

[15] S. Abe, Maximum-power quantum-mechanical Carnot engine, Phys. Rev. E 83, 041117 (2011).

[16] J. Wang, J. He, and Z. Wu, Efficiency at maximum power output of quantum heat engines under finite-time operation, Phys. Rev. E 85, 031145 (2012).

[17] A. E. Allahverdyan, K. V. Hovhannisyan, A. V. Melkikh, and S. G. Gevorkian, Carnot Cycle at Finite Power: Attainability of Maximal Efficiency, Phys. Rev. Lett. 111, 050601 (2013).

[18] R. D. Dann and R. Kosloff, Quantum signatures in the quantum Carnot cycle, New J. Phys. 22, 013055 (2020).

[19] P. Abiuso and M. Perarnau-Llobet, Optimal Cycles for LowDissipation Heat Engines, Phys. Rev. Lett. 124, 110606 (2020)

[20] U. Seifert, Stochastic thermodynamics, fluctuation theorems and molecular machines, Rep. Prog. Phys. 75, 126001 (2012).

[21] G. Verley, M. Esposito, T. Willaert, and C. Van den Broeck, The unlikely Carnot efficiency, Nat. Commun. 5, 4721 (2014).

[22] G. Verley, T. Willaert, C. Van den Broeck, and M. Esposito, Universal theory of efficiency fluctuations, Phys. Rev. E 90, 052145 (2014).

[23] M. Polettini, G. Verley, and M. Esposito, Efficiency Statistics at All Times: Carnot Limit at Finite Power, Phys. Rev. Lett. 114, 050601 (2015).
[24] S. K. Manikandan, L. Dabelow, R. Eichhorn, and S. Krishnamurthy, Efficiency Fluctuations in Microscopic Machines, Phys. Rev. Lett. 122, 140601 (2019).

[25] V. Holubec, An exactly solvable model of a stochastic heat engine: optimization of power, power fluctuations and efficiency, J. Stat. Mech. (2014) P05022.

[26] V. Holubec and A. Ryabov, Work and power fluctuations in a critical heat engine, Phys. Rev. E 96, 030102(R) (2017).

[27] P. Pietzonka and U. Seifert, Universal Trade-Off between Power, Efficiency and Constancy in Steady-State Heat Engines, Phys. Rev. Lett. 120, 190602 (2018).

[28] V. Holubec and A. Ryabov, Cycling Tames Power Fluctuations near Optimum Efficiency, Phys. Rev. Lett. 121, 120601 (2018).

[29] C. J. Foot, Atomic Physics (Oxford University Press, Oxford, UK, 2005).

[30] R. L. Brooks, The Fundamentals of Atomic and Molecular Physics (Springer, Berlin, 2013).

[31] P. M. Chaikin and T. C. Lubensky, Principles of Condensed Matter Physics (Cambridge University Press, Cambridge, UK, 2000).

[32] V. Bazani, A. Credi, and M. Venturi, Molecular Devices and Machines (Wiley-VCH, Weinheim, 2003).

[33] S. J. Blundell and K. M. Blundell, Concepts in Thermal Physics (Oxford University Press, Oxford, UK, 2006).

[34] M. Evangelisti, F. Luis, L. J. de Jongh, and M. Affronte, Magnetothermal properties of molecule-based materials, J. Mater. Chem. 16, 2534 (2006).

[35] B. Everitt, The Cambridge Dictionary of Statistics (Cambridge University Press, Cambridge, UK, 1998).

[36] V. Gritsev, P. Barmettler, and E. Demler, Scaling approach to quantum non-equilibrium dynamics of many-body systems, New J. Phys. 12, 113005 (2010).

[37] C. Jarzynski, Generating shortcuts to adiabaticity in quantum and classical dynamics, Phys. Rev. A 88, 040101(R) (2013).

[38] A. del Campo, Shortcuts to Adiabaticity by Counterdiabatic Driving, Phys. Rev. Lett. 111, 100502 (2013).

[39] S. Deffner, C. Jarzynski, and A. del Campo, Classical and Quantum Shortcuts to Adiabaticity for Scale-Invariant Driving, Phys. Rev. X 4, 021013 (2014).

[40] M. Beau, J. Jaramillo, and A. del Campo, Scaling-up quantum heat engines efficiently via shortcuts to adiabaticity, Entropy 18, 168 (2016).

[41] See Supplemental Material at http://link.aps.org/supplemental/ 10.1103/PhysRevResearch.3.L032041 for details about the derivation of the work distribution and of the coefficients of variation of work.

[42] P. Talkner, E. Lutz, and P. Hänggi, Fluctuation theorems: Work is not an observable, Phys. Rev. E 75, 050102(R) (2007).

[43] A. E. Allahverdyan, R. S. Johal, and G. Mahler, Work extremum principle: Structure and function of quantum heat engines, Phys. Rev. E 77, 041118 (2008).

[44] D. Gelbwaser-Klimovsky, W. Niedenzu, P. Brumer, and G. Kurizki, Power enhancement of heat engines via correlated thermalization in a three-level "working fluid", Sci. Rep. 5, 14413 (2015).

[45] W. Niedenzu, D. Gelbwaser-Klimovsky, and G. Kurizki, Performance limits of multilevel and multipartite quantum heat machines, Phys. Rev. E 92, 042123 (2015). 
[46] H. T. Quan, P. Zhang, and C. P. Sun, Quantum heat engine with multilevel quantum systems, Phys. Rev. E 72, 056110 (2005).

[47] J. Wang and J. He, Optimization on a three-level heat engine working with two noninteracting fermions in a one-dimensional box trap, J. Appl. Phys. 111, 043505 (2012).

[48] R. Wang, J. Wang, J. He, and Y. Ma, Performance of a multilevel quantum heat engine of an ideal $N$-particle Fermi system, Phys. Rev. E 86, 021133 (2012). 